\title{
Amygdalin inhibits renal fibrosis in chronic kidney disease
}

\author{
JUNQI GUO, WEIZHENG WU, MINGXIONG SHENG, SHUNLIANG YANG and JIANMING TAN \\ Organ Transplant Institute, Fuzhou General Hospital, \\ Dong Fang Hospital of Xiamen University and Fujian Key Laboratory of Transplant Biology, \\ Fuzhou, Fujian 350025, P.R. China
}

Received December 23, 2012; Accepted February 18, 2013

DOI: $10.3892 / \mathrm{mmr} .2013 .1391$

\begin{abstract}
Renal interstitial fibrosis is a common outcome of chronic renal diseases. Amygdalin is one of a number of nitrilosides, the natural cyanide-containing substances abundant in the seeds of plants of the prunasin family that are used to treat cancer and relieve pain. However, whether amygdalin inhibits the progression of renal fibrosis or not remains unknown. The present study aimed to assess the therapeutic potential of amygdalin by investigating its effect and potential mechanism on the activation of renal interstitial fibroblast cells and renal fibrosis in rat unilateral ureteral obstruction (UUO). Treatment of the cultured renal interstitial fibroblasts with amygdalin inhibited their proliferation and the production of transforming growth factor (TGF)- $\beta 1$. In the rat model of obstructive nephropathy, following ureteral obstruction, the administration of amygdalin immediately eliminated the extracellular matrix accumulation and alleviated the renal injury on the 21st day. Collectively, amygdalin attenuated kidney fibroblast (KFB) activation and rat renal interstitial fibrosis. These results indicate that amygdalin is a potent antifibrotic agent that may have therapeutic potential for patients with fibrotic kidney diseases.
\end{abstract}

\section{Introduction}

The pathogenesis of the majority of chronic kidney diseases (CKDs) involves a complex mechanism of hemodynamic and inflammatory processes that leads to renal fibrosis and tubulointerstitial scarring, with subsequent progression towards end-stage renal disease (ESRD) (1). Tubulointerstitial fibrosis is the final common pathway in late-stage renal disease. The

Correspondence to: Dr Jianming Tan, Fuzhou General Hospital, Xiamen University, 156 Xi Er Huang Road, Fuzhou, Fujian 350025, P.R. China

E-mail: tanjm156@yahoo.com.cn

Abbreviations: CKD, chronic kidney disease; UUO, unilateral ureteral obstruction; ECM, extracellular matrix; TGF- $\beta$, transforming growth factor- $\beta$; KFB, kidney fibroblast

Key words: amygdalin, renal interstitial fibrosis, fibroblast proliferation, TGF- $\beta 1$, unilateral ureteral obstruction pathogenesis of kidney fibrosis is characterized by the overproduction and deposition of extracellular matrix (ECM), which ultimately leads to fibrotic lesions and tissue scarring $(2,3)$. Renal interstitial fibroblasts are the principal effector cells responsible for ECM overproduction in the fibrotic kidney and their activation is regarded as a key event in the pathogenesis of chronic renal fibrosis (4). It is well-known that the upregulation of transforming growth factor (TGF)- $\beta 1$ signaling is considered to be a convergent pathway following renal injury, irrespective of the initial etiologies (5). An increase in the production of TGF- $\beta$ is one of the most important mechanisms in the pathogenesis of renal fibrogenesis (6). TGF- $\beta 1$ induces renal fibrosis by activating interstitial fibroblasts, causing them to produce large amounts of matrix components. These actions lead to glomerulosclerosis and tubulointerstitial fibrosis and ultimately to ESRD (7).

Studies have shown that therapeutic interventions, including the blockade of the renin-angiotensin-aldosterone system and the use of immunosuppressive drugs retard the progression of renal disease in experimental models (8) and human CKD clinical trials $(9,10)$. Although these strategies promote renoprotective effects, they fail to arrest the progression of renal fibrosis and scarring. Considering the fact that interstitial fibrosis represents the final common pathway of CKD, a therapeutic intervention with drugs that exhibit antifibrotic properties may be an attractive choice of therapy for arresting the autonomous fibrogenic process in chronic progressive nephropathies.

Amygdalin (vitamin B17; previously known as Laetrile) is one of a number of nitrilosides, the natural cyanide-containing substances abundant in the seeds of the prunasin family, including apricots, almonds, peaches, apples and other rosaceous plants. Among the prunasins, armeniacae semen has been used for the treatment of asthma, bronchitis, emphysema, leprosy, colorectal cancer, leucoderma and pain (11). Amygdalin is composed of two molecules of glucose, one of which is benzaldehyde, which induces an anti-neoplastic compound. Amygdalin has also been used to treat cancers and relieve pain (12).

In the present study, the effect of amygdalin on the kidney fibroblast (KFB) activation in normally cultured rat KFBs and its therapeutic potential on renal fibrosis in animal models of unilateral ureteral obstruction (UUO) was examined. Furthermore, the mechanism by which amygdalin inhibits renal fibroblast activation and fibrogenesis was investigated. 
The results suggested that amygdalin is a potent drug that can be used to reduce renal fibrosis during CKD progression and that its therapeutic mechanism, at least in part, blocks interstitial fibroblast cell activation.

\section{Materials and methods}

Separation and culture of the KFBs. A cell culture experiment was performed using renal fibroblasts obtained from kidney explants. For the primary culture, kidneys from rats were surgically and aseptically removed. Kidney sections $\left(1 \mathrm{~mm}^{3}\right)$ were seeded in $25-\mathrm{cm}^{2}$ bottles and cultured in Dulbecco's modified Eagle's medium (DMEM; Gibco Corp., Carlsbad, CA, USA), supplemented with $20 \%$ fetal calf serum (FCS; Cultilab, Campinas, Brazil) and antibiotics (amphotericin, $2.5 \mathrm{mg} / \mathrm{ml}$; ampicillin, $100 \mathrm{mg} / \mathrm{ml}$; and streptomycin, $100 \mathrm{mg} / \mathrm{ml}$; Gibco Corp.), at $37^{\circ} \mathrm{C}$ in a humidified atmosphere of $5 \% \mathrm{CO}_{2}$. When the cell outgrowth from the explants was initiated, the remaining tissue was removed. Once the cells reached confluence, they were harvested and split in a ratio of 1:3. Subsequent to 4-6 passages, the cells exhibited a typical fibroblast morphology. The cells were phenotypically characterized on the basis of their immunocytochemistry. The following antibodies were used: mouse anti-vimentin, mouse anti-desmin and mouse anti-keratin (Sigma Aldrich, St. Louis, MO, USA).

Immunocytochemical staining analysis. Cells were grown on glass coverslips. Following treatment, the cells were fixed with 4\% paraformaldehyde for $20 \mathrm{~min}$ and permeablilized with $1 \%$ Triton X-100 for $10 \mathrm{~min}$ at room temperature. Subsequent to further washing, the cells were blocked with $10 \%$ goat serum for $30 \mathrm{~min}$ at room temperature. The cells were then incubated with the primary antibodies at room temperature for $2 \mathrm{~h}$ followed by incubation with the secondary antibody for an additional $1 \mathrm{~h}$. The slides were stained with 3,3'-diaminobenzidine (DAB) and the nuclei were counterstained with hematoxylin for $10 \mathrm{~min}$. The coverslips were mounted on glass slides with anti-fade mounting media (Invitrogen, Carlsbad, CA, USA) and the images were visualized using a Zeiss LSM 710 laser confocal fluorescence microscope (Zeiss, Oberkochen, Germany).

3-(4,5-Dimethylthiazol-2-yl)-2,5-diphenyltetrazolium bromide (MTT) assay. The cells were grown in a final volume of $100 \mu \mathrm{l}$ medium per well in 96-well plates. To determine the antiproliferative effect of amygdalin, the cells were treated with amygdalin at concentrations of 25, 50, 80, 100 and $200 \mu \mathrm{g} /$ $\mathrm{ml}$ for $24 \mathrm{~h}$. The cells of the control group were left untreated. Subsequent to adding $10 \mu \mathrm{l}$ MTT labeling reagent, containing $5 \mathrm{mg} / \mathrm{ml} \mathrm{MTT}$ in phosphate-buffered saline (PBS), to each well, the plates were incubated for $4 \mathrm{~h}$. The optical density (OD) was calculated as the difference between the absorbance at the reference wavelength and that observed at the test wavelength. Inhibition ratio was calculated as: (1 - OD value in medication group / OD value in control group) x 100 .

TGF- $\beta 1$ enzyme-linked immunosorbent assay (ELISA). Human peripheral blood mononuclear cells (PBMC) were obtained through common separation at a concentration of $1 \times 10^{6}$ cells $/ \mathrm{ml}$. Besides the control group, the cells in the treatment groups were treated with amygdalin at concentrations of 25, 50, 100, 200, 400 and $800 \mu \mathrm{g} / \mathrm{ml}$. The cells were cultured for $48 \mathrm{~h}$ and the total TGF- $\beta 1$ was measured in the cell culture supernatant using a commercial sandwich ELISA kit according to the manufacturer's instructions.

\section{UUO rat kidney model and amygdalin treatment}

Construction of the UUO model. The UUO model was established in male, 180-200 $\mathrm{g}$ Wistar rats as described in a previous study, with minor modifications (13). Briefly, the abdominal cavity was exposed via a midline incision and the left ureter was isolated and ligated. The contralateral kidney was used as a control. To examine the efficacy of amygdalin in renal fibrosis subsequent to UUO injury, various concentrations of amygdalin ( 3 and $5 \mathrm{mg} / \mathrm{kg} /$ day) were intraperitoneally injected immediately following ureteral ligation. The animals were sacrificed and the kidneys removed at days 7, 14 and 21.

Pathological examination. Following anesthetization, the kidneys of the animals were removed for pathological examination using Masson's trichrome staining to identify the interstitial collagen with a blue coloration. The evaluation scope of each parameter was 0-3 and the evaluation score of each tubulointerstitial sample was 0-9. Masson's staining of each kidney tissue sample was evaluated by three observers using a blind method to obtain a mean value.

Statistical analysis. Values were expressed as the mean \pm SD for each group. Statistical differences between two groups were analyzed by the unpaired Student's t-test and differences between multiple groups of data were analyzed by a one-way ANOVA with a Bonferroni correction. $\mathrm{P}<0.05$ was considered to indicate a statistically significant difference.

\section{Results}

\section{Impact of amygdalin on the proliferation of the KFBs}

Cell characterization. The cells were cultured and reached confluence after 3-4 days (Fig. 1). The cells were characterized by their morphological appearance under phase contrast microscopy and by the positive staining for vimentin and the negative staining for keramin and desmin. This demonstrated that the cells were KFBs.

Effect of amygdalin on the cell proliferation in the KFBs. As is evident in Fig. 2, with the extension of the culture period, the cell proliferation was significantly enhanced in the control group. Cell proliferation was significantly decreased in the amygdalin group compared with the control group following $48 \mathrm{~h}$ and $72 \mathrm{~h}$ of treatment $(\mathrm{P}<0.05)$. However, there was no significant difference between the control and amygdalin groups subsequent to $24 \mathrm{~h}$ of treatment.

In addition, the antiproliferative effect of amygdalin was observed at a concentration of $25-400 \mu \mathrm{g} / \mathrm{ml}$ and the most potent inhibition was observed at a concentration of $100 \mu \mathrm{g} /$ $\mathrm{ml}$, with an inhibition rate of $31.53 \%$ (Fig. 3).

Effect of amygdalin on the expression of TGF- $\beta 1$ produced by human lymphocytes. As TGF- $\beta 1$ is a major cytokine that is able to induce the transformation of quiescent renal fibroblasts to myofibroblasts, the present study first examined the effect of amygdalin on TGF- $\beta 1$ production. Fig. 4 shows that when 

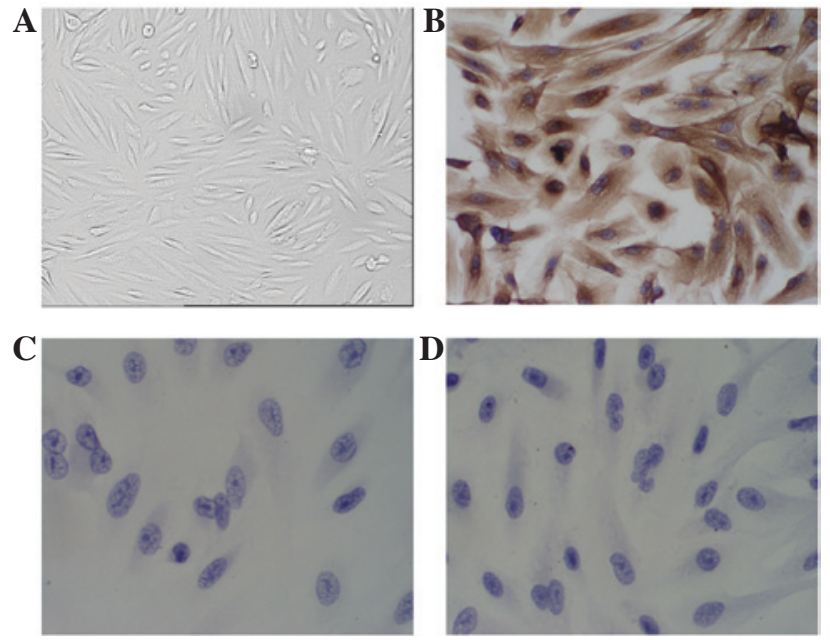

Figure 1. Immunocytochemistry for the phenotypic characterization of the fibroblasts obtained from the primary culture of kidney explants. (A) The slides incubated without the primary antibody were used as the negative control. The cells derived from the kidney explants were positive for (B) vimentin and negative for (C) keramin and (D) desmin.

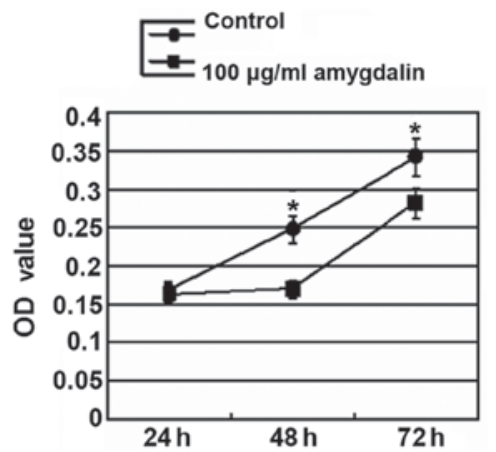

Figure 2. Time course of the inhibition of the renal fibroblast proliferation by amygdalin. MTT assay of the fibroblasts from the primary culture. The cells were treated with amygdalin for 24,48 and $72 \mathrm{~h}$ at a concentration of $100 \mu \mathrm{g} / \mathrm{ml} .{ }^{*} \mathrm{P}<0.05$ vs. control group. MTT, 3-(4,5-Dimethylthiazol-2-yl)2,5-diphenyltetrazolium bromide; OD, optical density.

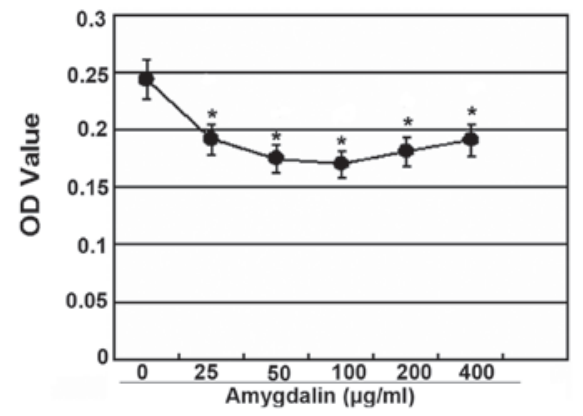

Figure 3. Dose-dependent inhibition of the renal fibroblast proliferation by amygdalin. MTT assay of the fibroblasts from the primary culture. The cells were treated with amygdalin for $48 \mathrm{~h}$ at the indicated concentrations. ${ }^{*} \mathrm{P}<0.05$ vs. control group. MTT, 3-(4,5-dimethylthiazol-2-yl)-2,5-diphenyltetrazolium bromide; OD, optical density.

within the concentration range of 50-400 $\mu \mathrm{g} / \mathrm{ml}$, amygdalin suppressed the TGF- $\beta 1$ secretion in the peripheral blood lymphocytes, which was stimulated by PHA, in a concen-

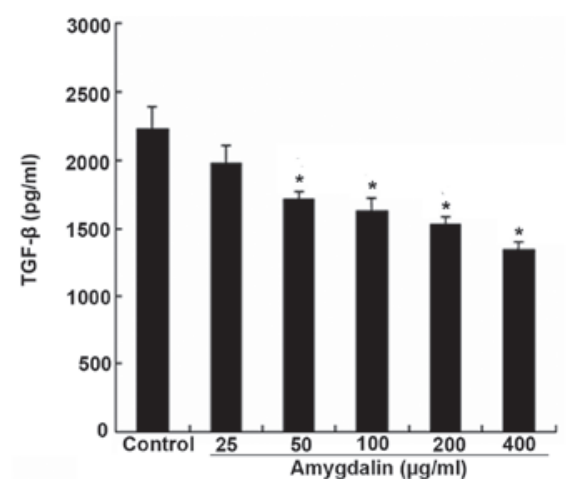

Figure 4. Effect of amygdalin on the TGF- $\beta 1$ production in the cultured fibroblasts. The concentration of the TGF- $\beta 1$ protein in the supernatant was measured subsequent to $48 \mathrm{~h}$ of the primary culture of the renal fibroblasts. ${ }^{*} \mathrm{P}<0.05$ vs. control group.
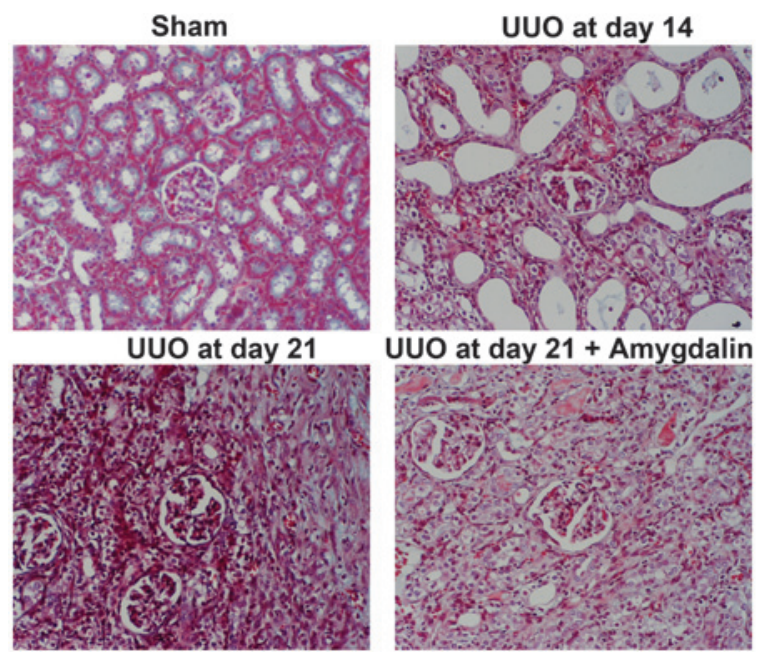

Figure 5. Photomicrographs of Masson trichrome staining of kidney tissue subsequent to various treatments. Amygdalin attenuates the deposition of the ECM in the obstructed rat kidneys. UUO, unilateral ureteral obstruction; ECM, extracellular matrix.

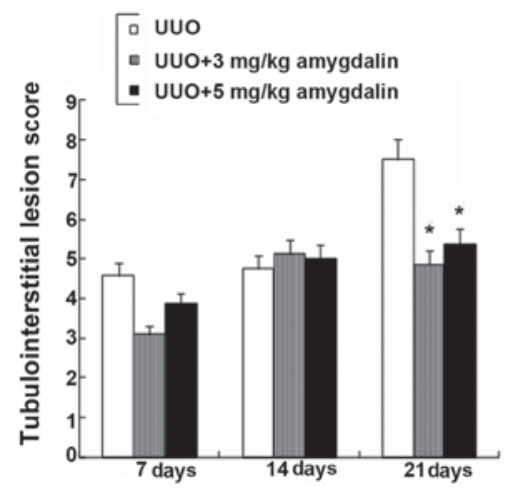

Figure 6. Effect of amygdalin on renal interstitial fibrosis in the rat. The extent of the rat tubulointerstitial lesions (TIL) with the treatment of amygdalin ( 3 and $5 \mathrm{mg} / \mathrm{kg} / \mathrm{day}$ ). ${ }^{*} \mathrm{P}<0.05$ vs. control group. UUO, unilateral ureteral obstruction.

tration-dependent manner $(\mathrm{P}<0.05)$. These data collectively indicate that amygdalin is a potent agent for blocking the activation of cultured renal interstitial fibroblasts. 
Amygdalin ameliorated renal fibrosis in the obstructed kidney. As the major feature of renal fibrosis is the increased levels of ECM, the present study examined the effect of amygdalin on the expression of interstitial collagen fibrils, using Masson trichrome staining to investigate the ability of amygdalin in suppressing myofibroblast activation in vivo. As shown in Fig. 5, the kidneys with ureteral obstructions for 7 days exhibited severe morphological lesions characterized by tubular dilation with epithelial atrophy and interstitial expansion with collagen accumulation and deposition, as evidenced by an increase in the trichrome-positive areas within the tubulointerstitium subsequent to UUO injury. By contrast, the kidneys from the rats administered with amygdalin exhibited a marked attenuation of these morphological lesions, with less fibrosis in the interstitium. These data showed an efficacy of amygdalin for inhibiting the accumulation of the ECM proteins following obstructive injury.

Fig. 6 shows the tubulointerstitial lesions (TILs) in the experimental rats were aggravated on day 21 . However, the extent of the rat TILs in the $3 \mathrm{mg}$ and $5 \mathrm{mg}$ amygdalin treatment groups was significantly reduced on day $21(\mathrm{P}<0.05)$. In addition, there was no significant difference in the TILs among the three groups on the 7th and 14th days ( $>>0.05)$. Amygdalin is therefore effective in preventing renal fibrosis progression in rats following UUO injury.

\section{Discussion}

Renal interstitial fibrosis is the final result of TILs, which have various causes, and also one of the major reasons behind final-stage renal failure (14). Modern pharmacology indicates that Traditional Chinese Medicine shows promising application prospects for the prevention and treatment of renal fibrosis and has consequently drawn the attention of investigators. Amygdalin is an aromatic cyanogenic glycoside that exists in the seeds of rosaceous plants, including armeniaca, wild apricot, peach, mountain peach and plum. The structural formula of amydalin is (6-O- $\beta$-D-glucopyranosyl- $\beta$-D-glucopyranosyl) oxy] (phenyl) acetonitrile (15). In the present study, fibroblasts were successfully separated from the rat kidneys by a digestive method and then identified by a immunocytochemical method. Notably, with the extension of the culture period, significant cell proliferation was observed in the control group; the proliferative levels in the amygdalin groups at the varying concentrations were significantly lower than those of the control group. Amygdalin was able to suppress the proliferative activity of the fibroblasts in a concentration-dependent manner. Furthermore, the suppressive effect of amygdalin reached a peak subsequent to $48 \mathrm{~h}$ of incubation at a concentration of $100 \mu \mathrm{g} / \mathrm{ml}$. However, the detailed mechanism by which amygdalin suppresses KFB proliferation has yet to be fully investigated. Fibroblasts play a significant role in the physiological and pathological processes of renal fibrosis, and the treatment countermeasures that have used fibroblasts as the target cells have shown the corresponding effects (16).

In the present study, amygdalin was able to suppress TGF- $\beta 1$ secretion in the lymphocytes at concentrations of $25-400 \mu \mathrm{g} / \mathrm{ml}$. As an important profibrogenic cytokine, TGF- $\beta 1$ plays a pivotal role in the occurrence, development and other links of renal interstitial fibrosis (7). Furthermore, it has been demonstrated that the induction and activation of TGF- $\beta 1 / \mathrm{Smad}$ is essential for eliciting the fiber formation reaction; equally important is the loss of the Smad antagonist, which may cause the fiber formation signal to become out of control $(17,18)$. In the pathogenesis of renal interstitial fibrosis, fibroblasts and myofibroblasts are the major source of TGF- $\beta 1$ production. The cytological basis for renal interstitial fibrosis is the activation of myofibroblasts, which may come from various resources. With the exception of the fibroblasts inherent in the kidney, a fairly large number of myofibroblasts are from the renal tubular epithelial cells. Specifically, renal tubular epithelial cells may transform into myofibroblasts through the mechanism of epithelial-myofibroblast transdifferentiation (EMT) $(19,20)$, resulting in a large quantity of ECM and promoting the occurrence of fibrosis. TGF- $\beta 1$ plays a significant role in the EMT process (21). The present study also demonstrated that amygdalin exerted an antifibrotic effect through the inhibition of TGF- $\beta 1$ secretion in the lymphocytes.

Based on in vivo results that showed that amygdalin was able to suppress KFB proliferation, the present study further investigated the antifibrotic effect of amygdalin on renal interstitial fibrosis by means of UUOs. The pathological changes of the kidney tissues in the obstruction process and the effect of amygdalin treatment on the damage in obstructed kidneys were examined. Renal interstitial fibrosis is characterized by the accumulation and increase of renal interstitial cells and collagen components, in combination with renal tubular fibrosis or expansion and deformation (22). At seven days post-UUO surgery, inflammatory cell infiltration, cell proliferation, renal tubular expansion and other pathological changes in the kidney tissues were observed. Thereafter the kidney tissues showed progressive renal tubular fibrosis and renal interstitial fibrosis. Following UUO surgery, early-stage renal interstitial fibrosis damage had already occurred in the obstructed side of the kidney on the 7th day. Although renal interstitial fibrosis had become fairly severe on the 21 st day, there were no significant pathological changes to the glomerulus, as in accordance with previous studies $(14,23)$. This confirmed the characteristics for the pathological change in the injured kidney. Furthermore, on the 21st day, in comparison with the rats in the obstruction group, the extent of the rat tubulointerstitial lesions in the amygdalin treatment groups was significantly reduced. Consequently, amygdalin treatment may significantly alleviate the extent of the pathological damage to the kidney and postpone the process of renal interstitial fibrosis.

In summary, the present study has demonstrated that amygdalin was able to suppress KFB proliferation and TGF- $\beta 1$ secretion in the lymphocytes and thus was able to significantly alleviate the extent of the UUO pathological damage to the kidney and postpone the process of renal interstitial fibrosis, which further accounts for the anti-fibrotic effect of amygdalin. Although the detailed mechanisms behind the action of amygdalin remain undefined, we hypothesize that the mechanisms may be involved in increasing the secretion of type I collagenase, inhibiting KFB proliferation, accelerating apoptosis and suppressing type I collagen synthesis. However, future studies are required to investigate the mechanisms by which amygdalin protects against renal interstitial fibrosis. 


\section{Acknowledgements}

This study is supported by grants from the Key Laboratory of Fujian Province (No. 2008J1006) and the Nanjing Medical Technology Innovation Project, China (No. 2009MA093).

\section{References}

1. Noronha IL, Fujihara CK and Zatz R: The inflammatory component in progressive renal disease - are interventions possible? Nephrol Dial Transplant 17: 363-368, 2002.

2. Wynn TA: Cellular and molecular mechanisms of fibrosis. J Pathol 214: 199-210, 2008.

3. Neilson EG: Mechanisms of disease: Fibroblasts - a new look at an old problem. Nat Clin Pract Nephrol 2: 101-108, 2006.

4. Boor P, Ostendorf T and Floege J: Renal fibrosis: novel insights into mechanisms and therapeutic targets. Nat Rev Nephrol 6 : 643-656, 2010

5. Liu Y: Renal fibrosis: new insights into the pathogenesis and therapeutics. Kidney Int 69: 213-217, 2006.

6. Eddy AA: Molecular basis of renal fibrosis. Pediatr Nephrol 15 : 290-301, 2000

7. Böttinger EP and Bitzer M: TGF-beta signaling in renal disease. J Am Soc Nephrol 13: 2600-2610, 2002.

8. Fujihara CK, Malheiros DM, Zatz R and Noronha IL: Mycophenolate mofetil attenuates renal injury in the rat remnant kidney. Kidney Int 54: 1510-1519, 1998.

9. Lewis EJ, Hunsicker LG, Clarke WR, et al; Collaborative Study Group: Renoprotective effect of the angiotensin-receptor antagonist irbesartan in patients with nephropathy due to type 2 diabetes. N Engl J Med 345: 851-860, 2001

10. Brenner BM, Cooper ME, de Zeeuw D, et al; RENAAL Study Investigators: Effects of losartan on renal and cardiovascular outcomes in patients with type 2 diabetes and nephropathy. $\mathrm{N}$ Engl J Med 345: 861-869, 2001.

11. Chang HK, Yang HY, Lee TH, et al: Armeniacae semen extract suppresses lipopolysaccharide-induced expressions of cyclooxygenase (correction of cycloosygenase)-2 and inducible nitric oxide synthase in mouse BV2 microglial cells. Biol Pharm Bull 28: 449-454, 2005.
12. Fukuda $T$, Ito $H$, Mukainaka $T$, Tokuda $H$, Nishino $H$ and Yoshida T: Anti-tumor promoting effect of glycosides from Prunus persica seeds. Biol Pharm Bull 26: 271-273, 2003.

13. Liu N, Tolbert E, Pang M, Ponnusamy M, Yan H and Zhuang S: Suramin inhibits renal fibrosis in chronic kidney disease. J Am Soc Nephrol 22: 1064-1075, 2011.

14. Robertson H, Ali S, McDonnell BJ, Burt AD and Kirby JA: Chronic renal allograft dysfunction: the role of T cell-mediated tubular epithelial to mesenchymal cell transition. J Am Soc Nephrol 15: 390-397, 2004.

15. Chang HK, Shin MS, Yang HY, et al: Amygdalin induces apoptosis through regulation of $\mathrm{Bax}$ and $\mathrm{Bcl}-2$ expressions in human DU145 and LNCaP prostate cancer cells. Biol Pharm Bull 29: $1597-1602,2006$

16. Vongwiwatana A, Tasanarong A, Rayner DC, Melk A and Halloran PF: Epithelial to mesenchymal transition during late deterioration of human kidney transplants: the role of tubular cells in fibrogenesis. Am J Transplant 5: 1367-1374, 2005.

17. Zeisberg M, Hanai J, Sugimoto $\mathrm{H}$, et al: BMP-7 counteracts TGF-beta1-induced epithelial-to-mesenchymal transition and reverses chronic renal injury. Nat Med 9: 964-968, 2003.

18. Wang W, Huang XR, Li AG, et al: Signaling mechanism of TGF-beta1 in prevention of renal inflammation: role of Smad7. J Am Soc Nephrol 16: 1371-1383, 2005.

19. Shihab FS, Bennett WM, Yi H and Andoh TF: Pirfenidone treatment decreases transforming growth factor-betal and matrix proteins and ameliorates fibrosis in chronic cyclosporine nephrotoxicity. Am J Transplant 2: 111-119, 2002.

20. Liu Y: Epithelial to mesenchymal transition in renal fibrogenesis pathologic significance, molecular mechanism, and therapeutic intervention. J Am Soc Nephrol 15: 1-12, 2004.

21. Heeg MH, Koziolek MJ, Vasko R, et al: The antifibrotic effects of relaxin in human renal fibroblasts are mediated in part by inhibition of the Smad2 pathway. Kidney Int 68: 96-109, 2005.

22. Satoh S, Yamaguchi T, Hitomi A, et al: Fasudil attenuates interstitial fibrosis in rat kidneys with unilateral ureteral obstruction. Eur J Pharmacol 455: 169-174, 2002.

23. Strutz F and Müller GA: Renal fibrosis and the origin of the renal fibroblast. Nephrol Dial Transplant 21: 3368-3370, 2006. 